$19,10,11$

\title{
Электронные и теплофизические свойства газовых гидратов: результаты моделирования из первых принципов
}

\author{
(C) М.Б. Юнусов ${ }^{1}$, Р.М. Хуснутдинов ${ }^{1,2}$, А.В. Мокшин ${ }^{1,2}$ \\ ${ }^{1}$ Казанский (Приволжский) фредеральный университет, \\ Казань, Россия \\ ${ }^{2}$ Удмуртский фредеральный исследовательский центр УрО РАН, \\ Ижевск, Россия \\ E-mail: mukhammadbek@mail.ru
}

Поступила в Редакцию 23 сентября 2020 г.

В окончательной редакции 2 октября 2020 г.

Принята к публикации 2 октября 2020 г.

Представлены результаты ab initio молекулярно-динамического исследования электронных и теплофизических свойств гидрата метана с кубической структурой КС-І. Найдено хорошее согласие результатов моделирования для теплоемкости при постоянном объеме и плотности с экспериментальными данными. На основе анализа плотности электронных состояний определены температурные зависимости электронных свойств гидрата метана, включая уровень энергии Ферми, ширину и границы запрещенной зоны. Для пустого каркаса гидрата (водный клатратный каркас) рассчитаны спектры энергии электронов $E(\mathbf{k})$ вдоль направлений М-X, Х-Г, Г-М и Г-R. Установлено, что наличие молекул $\mathrm{CH}_{4}$ в водном клатрате приводит к увеличению энергии Ферми гидрата с 2.4 до $3.0 \mathrm{eV}$.

Ключевые слова: $a b$ initio вычисления, плотность электронных состояний.

DOI: 10.21883/FTT.2021.02.50485.203

\section{1. Введение}

Природные гидраты являются одной из форм существования газа в недрах Земли и перспективным источником углеводородного газа [1]. Гидраты природных газов или газовые гидраты - это нестехиометрические соединения, включения в которых молекулы газа (молекулы-гостя) заключены в полостях трехмерной решетки из молекул воды (каркас хозяина). Устойчивость водных клатратных каркасов, которые являются термодинамически менее стабильными, чем лед или жидкая вода при тех же условиях, обеспечивается за счет ван-дер-ваальсовых взаимодействий гость-хозяин [1-8]. Так, стабилизация структуры газового гидрата обеспечивается за счет отталкивания водного каркаса от внутренних молекул газа. В работе [9] с помощью квантово-механического моделирования в рамках обобщенно градиентного приближения детально исследовались структурная устойчивость и электронные свойства различных газовых гидратов $\left(\mathrm{CO}_{2}, \mathrm{CO}\right.$, $\mathrm{CH}_{4}$ и $\mathrm{H}_{2}$ ). Авторами было показано, что заполнение водного клатратного каркаса газом $\left(\mathrm{CO}_{2}, \mathrm{CO}, \mathrm{CH}_{4}\right.$ и $\mathrm{H}_{2}$ ) повышает стабильность структуры гидрата. Было установлено, что гидрат диоксида углерода является наиболее стабильным по сравнению с другими газовыми гидратами, энергия связи которого составила $-2.36 \mathrm{eV}$. Наименее стабильным оказался гидрат водорода с энергией связи $-0.36 \mathrm{eV}$. Энергия связи гидрата метана составила $-0.58 \mathrm{eV}$.

Структурные типы наиболее часто встречающихся газовых гидратов - это кубические структуры I и II, а также гексагональная структура III, обозначаемые как КС-I, КС-II и ГC-III, соответственно [2]. Гидрат метана преимущественно кристаллизуется в кубическую структуру, известную как тип КС-I, в которой как большие кластеры воды (с диаметром 5.86 $)$ ), так и малые кластеры воды (с диаметром $\sim 5.10 \AA$ ) могут улавливать молекулу метана, диаметр которой $\sim 4.36 \AA[10,11]$. Такая система обычно образуется, когда метан и вода вступают в контакт при температуре окружающей среды, т. е. $T \sim 300 \mathrm{~K}$ и умеренном давлении, $p \geq 0.6 \mathrm{MPa}[12]$. В настоящее время структурные особенности и теплофизические свойства газовых гидратов вызывают широкий интерес у исследователей. Это обусловлено, во-первых, возможностью использования гидратов природного газа в качестве нового источника углеводородного топлива. Согласно полученным оценкам, запасы газа в гидратах составляют $\sim 2 \cdot 10^{16} \mathrm{~m}^{3}$, что на порядки превосходит запасы обычного природного газа $[1,2]$. Во-вторых, в газовой отрасли остро стоит проблема гидратообразования в стволах скважин и газопроводах.

Цель настоящей работы заключается в $a b$ initio исследовании электронных и теплофизических свойств газовых гидратов с характерной кубической структурой КС-I.

\section{2. Детали вычислений}

Крупномасштабные молекулярно-динамические исследования из первых принципов гидрата метана с кубической структурой КС-I были выполнены в программном комплексе VASP $[13,14]$. На основе результатов рентгеноструктурного анализа Штакельберга и 

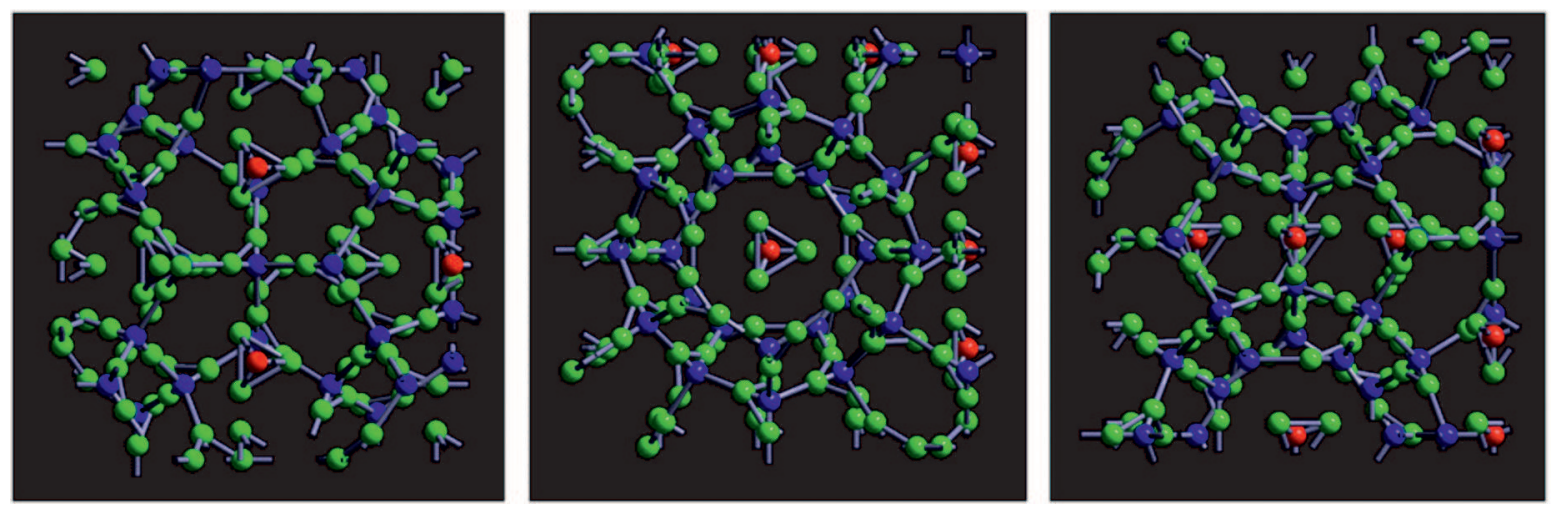

Рис. 1. (Цветной онлайн) Ячейка гидрата метана структуры КС-I в трех проекциях: красным, синим и зеленым цветами отмечены атомы углерода, кислорода и водорода соответственно.

Мюллера [15], Полинга и Марша [16], а также алгоритма оптимизации положений атомов водорода с помощью правил Бернала-Фаулера, и с учетом равенства нулю полного дипольного момента была сгенерирована кристаллическая структура клатратного гидрата КС-I. Гидрат метана образован через внедрение молекул $\mathrm{CH}_{4}$ в свободные полости каркаса водного клатрата. Моделируемая система имела размер $12 \times 12 \times 12 \AA$, которая включала 8 молекул метана и состояла из 178 ионов атомов водорода, углерода и кислорода. На рис. 1 представлена ячейка гидрата метана со структурой КС-I в трех проекциях. Моделирование выполнялось в изотермически-изохорическом (NVT)-ансамбле для температурной области $T=[200 ; 300] \mathrm{K}$ с шагом по температуре $\Delta T=20 \mathrm{~K}$. Для установления состояния термодинамического равновесия был применен термостат Нозе-Гувера. Во избежание нежелательных поверхностных явлений и эффекта конечномерной системы на ячейку моделирования по всем направлениям были наложены периодические граничные условия. Базисный набор состоял из плоских волн; электрон-ионное взаимодействие осуществлялось с помощью ультрамягких сглаженных псевдопотенциалов; обменно-корреляционная энергия была рассчитана в рамках обобщенноградиентного приближения $[13,14]$.

\section{3. Результаты и обсуждение}

Для проверки корректности результатов моделирования была рассчитана массовая плотность кристаллического гидрата метана. Полученное значение для исследуемой системы составило $\rho=918.4 \mathrm{~kg} / \mathrm{m}^{3}$, что находится в хорошем согласии с экспериментальным значением $\rho=910.0 \mathrm{~kg} / \mathrm{m}^{3}$ [17]. Была рассчитана температурная зависимость полной энергии системы для диапазона $T=[200 ; 300] \mathrm{K}$, которая, как оказалось, хорошо аппроксимируется линейной зависимостью вида

$$
E(T)=3.748 \cdot 10^{-21} T+1.374 \cdot 10^{-16}(\mathrm{~J}) .
$$

Средняя теплоемкость при постоянном объеме рассчитывалась по формуле

$$
C_{V}=\frac{1}{m} \frac{d Q}{d T}=\frac{1}{m}\left(\frac{d E}{d T}+p \frac{d V}{d T}-\mu \frac{d N}{d T}\right)=\frac{1}{m} \frac{d E}{d T}
$$

Полученное значение теплоемкости составило $C_{v}=2362.5 \mathrm{~J} /(\mathrm{kg} \cdot \mathrm{K})$, что хорошо согласуется с экспериментальными данными $C_{v}=2160 \pm 100 \mathrm{~J} /(\mathrm{kg} \cdot \mathrm{K})[18]$ и $2306 \mathrm{~J} /(\mathrm{kg} \cdot \mathrm{K})$ [19]. Для исследуемой системы была рассчитана плотность электронных состояний $N(E)$ с температурами из диапазона $T=[200 ; 300] \mathrm{K}$ с шагом $\Delta T=20 \mathrm{~K}$. Плотность электронных состояний и энергетические зоны для газового гидрата с кубической структурой КС-I при $T=200 \mathrm{~K}$ представлена на рис. 2 . Рассчитанные зависимости $N(E)$ находятся в хорошем качественном согласии с результатами квантово-механических расчетов для различных газовых гидратов $\left(\mathrm{CO}_{2}\right.$,

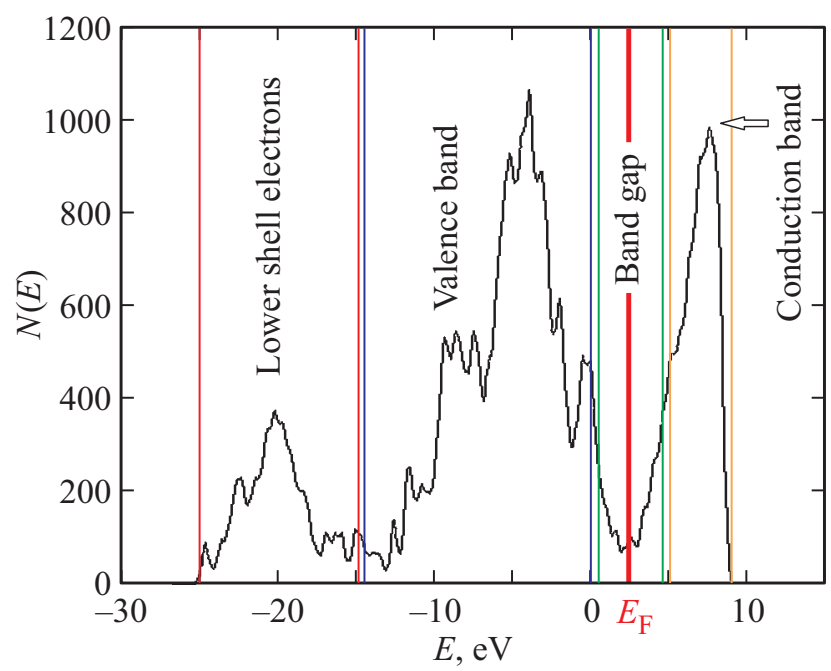

Pис. 2. (Цветной онлайн) Плотность электронных состояний и энергетические зоны для гидрата метана с кубической структурой КС-I при $T=200 \mathrm{~K}$. 

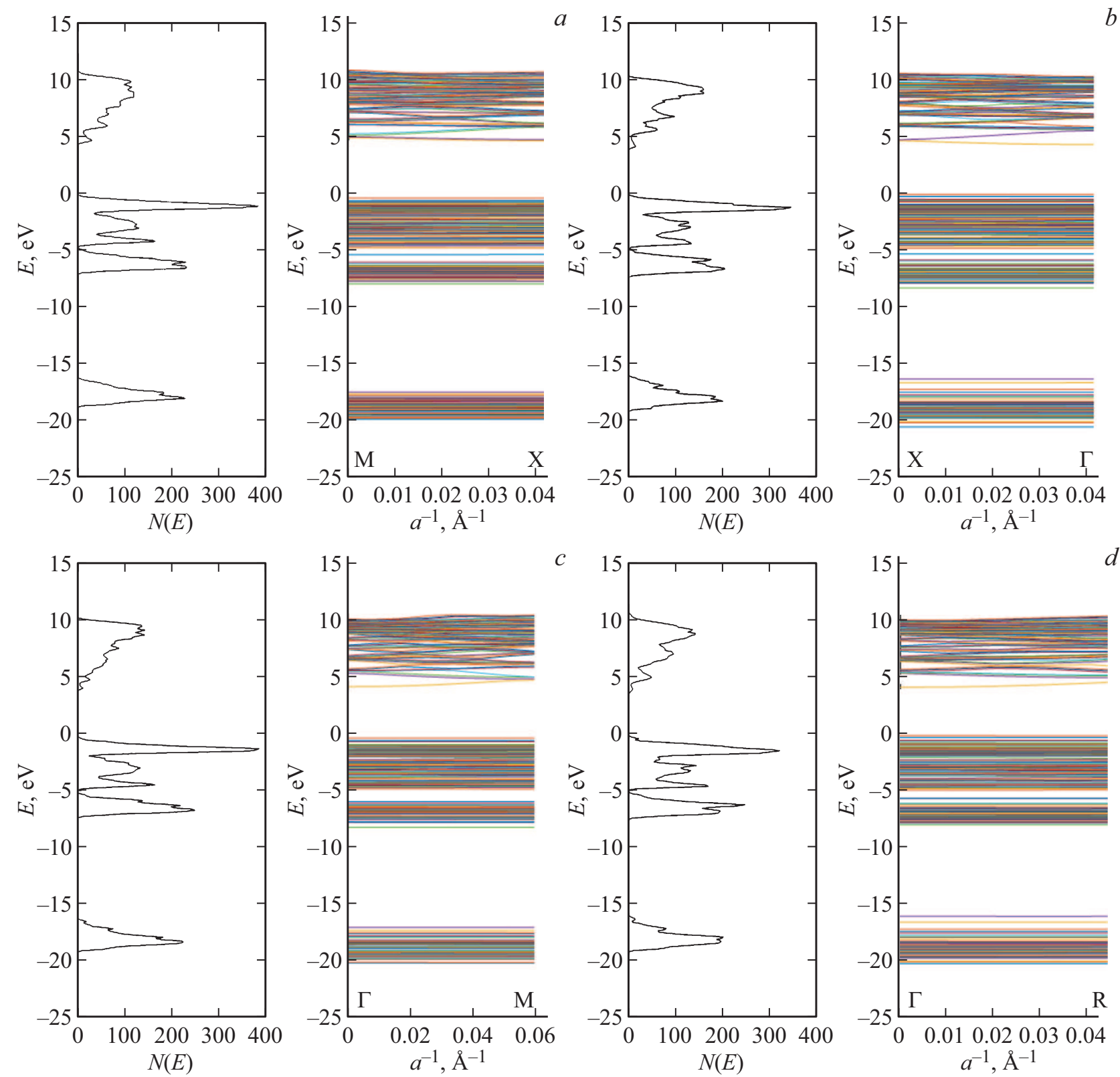

Pис. 3. (Цветной онлайн) Плотность электронных состояний и спектр энергии электронов $E(\mathbf{k})$ вдоль направлений: $a)$ М-Х; b) Х-Г; c) Г-M; d) Г-R. Ось абсцисс для зависимости $E(\mathbf{k})$ масштабирована на величину $a^{-1}$, где $a-$ параметр решетки $(a=12 \AA)$.

$\mathrm{CO}, \mathrm{CH}_{4}$ и $\left.\mathrm{H}_{2}\right)^{1}[9]$. Для определения границ запрещенной зоны была выполнена процедура подгонки формы контуров линий спектра гауссовыми функциями. Аппроксимация пиков плотности электронных состояний, прилегающих к запрещенной зоне, позволила найти приближенное значение энергии верхней границы валентной зоны $\left(E_{G} \max \right)$ и нижней границы зоны проводимости $\left(E_{G} \min \right)$. Положение уровня Ферми определялось как

$$
E_{F}=\frac{E_{G \max }+E_{G \min }}{2}+\frac{3}{4} k_{B} T \ln \left(\frac{m_{p}}{m_{n}}\right)
$$

\footnotetext{
${ }^{1}$ В работе [9] уровень энергии Ферми смещен влево по горизонтальной оси, т.е. $E_{F}=0 \mathrm{eV}$.
}

где $m_{p}$ и $m_{n}-$ эффективные массы дырок и электронов [20]. Вследствие малости второго слагаемого в (3), при расчете положений энергии уровня Ферми данный вклад не учитывался. Полученные значения для ширины $\Delta E_{G}$ и границ запрещенной зоны $\left(E_{G} \max , E_{G} \min \right)$, а также энергии уровня Ферми $E_{F}$ для гидрата метана с кубической структурой КС-I представлены в таблице.

Как видно из таблицы, значение энергии уровня Ферми с высокой точностью находится посередине запрещенной зоны. Полученные значения ширины щели $\Delta E_{G}$ между нижней границей зоны проводимости и верхней границей валентной зоны находятся в хорошем согласии с результатами квантово-механических расчетов для гидрата метана с кубической структурой КС-I 
Температурные зависимости ширины $\Delta E_{G}$ и границ запрещенной зоны $\left(E_{G \max }, E_{G \text { min }}\right)$, а также энергии Ферми $E_{F}$ для гидрата метана с кубической структурой КС-I

\begin{tabular}{c|c|c|c|c}
\hline$T, \mathrm{~K}$ & $E_{G \max }, \mathrm{eV}$ & $E_{G \min }, \mathrm{eV}$ & $E_{F}, \mathrm{eV}$ & $\Delta E_{G}, \mathrm{eV}$ \\
\hline 200 & 4.895 & 1.257 & 3.076 & 3.638 \\
220 & 4.897 & 1.091 & 3.039 & 3.896 \\
240 & 4.979 & 1.066 & 3.023 & 3.912 \\
260 & 5.090 & 1.471 & 3.281 & 3.620 \\
280 & 5.148 & 1.332 & 3.240 & 3.816 \\
300 & 5.310 & 1.302 & 3.306 & 4.009
\end{tabular}

при трех различных конфигурациях [21], обозначаемых как cI, cII и cIII. Значения $\Delta E_{G}$ для гидрата метана с конфигурациями cI, cII, сIII составляли, соответственно, $5.27,5.23$ и $3.81 \mathrm{eV}$.

Кроме того, для исследуемой системы обнаружено повышение энергии верхней и нижней границы запрещенной зоны с ростом температуры. В диапазоне температур 200-300 K проведена линейная аппроксимация значений энергии Ферми. При повышении температуры нижняя граница зоны проводимости поднимается со средней скоростью $d E_{G \max } / d T=0.0053 \mathrm{eV} / \mathrm{K}$. Верхняя граница валентной зоны поднимается со средней скоростью $d E_{G \min } / d T=0.0035 \mathrm{eV} / \mathrm{K}$. При этом энергия Ферми повышается со скоростью $d E_{F} / d T=0.0044 \mathrm{eV} / \mathrm{K}$. Разница в скорости роста границ запрещенной зоны приводит к изменению ее ширины. Средняя скорость ее расширения составила $d E_{G} / d T=0.0018 \mathrm{eV} / \mathrm{K}$.

Для каркаса гидрата КС-I с пустыми молекулярными полостями была рассчитана энергетическая зонная структура, которая представляет собой зависимость энергий электронов от волнового вектора $E(\mathbf{k})$ и которая дает представление о характере изменения энергии электрона при движении вдоль какого-либо направления в пространстве волновых векторов. Поскольку энергия электрона в кристалле является периодической функцией от $\mathbf{k}$, то при исследовании энергетического спектра электрона достаточно ограничиться рассмотрением волнового вектора $\mathbf{k}$ из диапазона $-\pi / a<k<\pi / a$, т. е. рассмотрением лишь первой зоны Бриллюэна. Как правило, в качестве выделенных направлений волнового вектора рассматриваются линии, соединяющие точки высокой симметрии. Гидрат КС-I характеризуется кубической решеткой, для которой зона Бриллюэна имеет кубическую форму. На рис. 3 представлена плотность электронных состояний и спектр энергии электронов $E(\mathbf{k})$ для каркаса гидрата КС-I с пустыми молекулярными полостями вдоль направлений: М-X; X-Г; Г-М и Г-R. Здесь центр зоны Бриллюэна обозначается символом $\Gamma$, центр грани - X, центр ребра - М и вершина - R.

На рис. 4 представлена энергетическая зонная структура кристалла гидрата с кубической структурой КC-I с пустыми молекулярными полостями. Как видно из рисунка, электроны нижних энергетических уровней $(-20 \div-17 \mathrm{eV})$ и валентной зоны $(-8 \div 0 \mathrm{eV})$ с большой точностью имеют прямую зависимость $E(\mathbf{k})$.

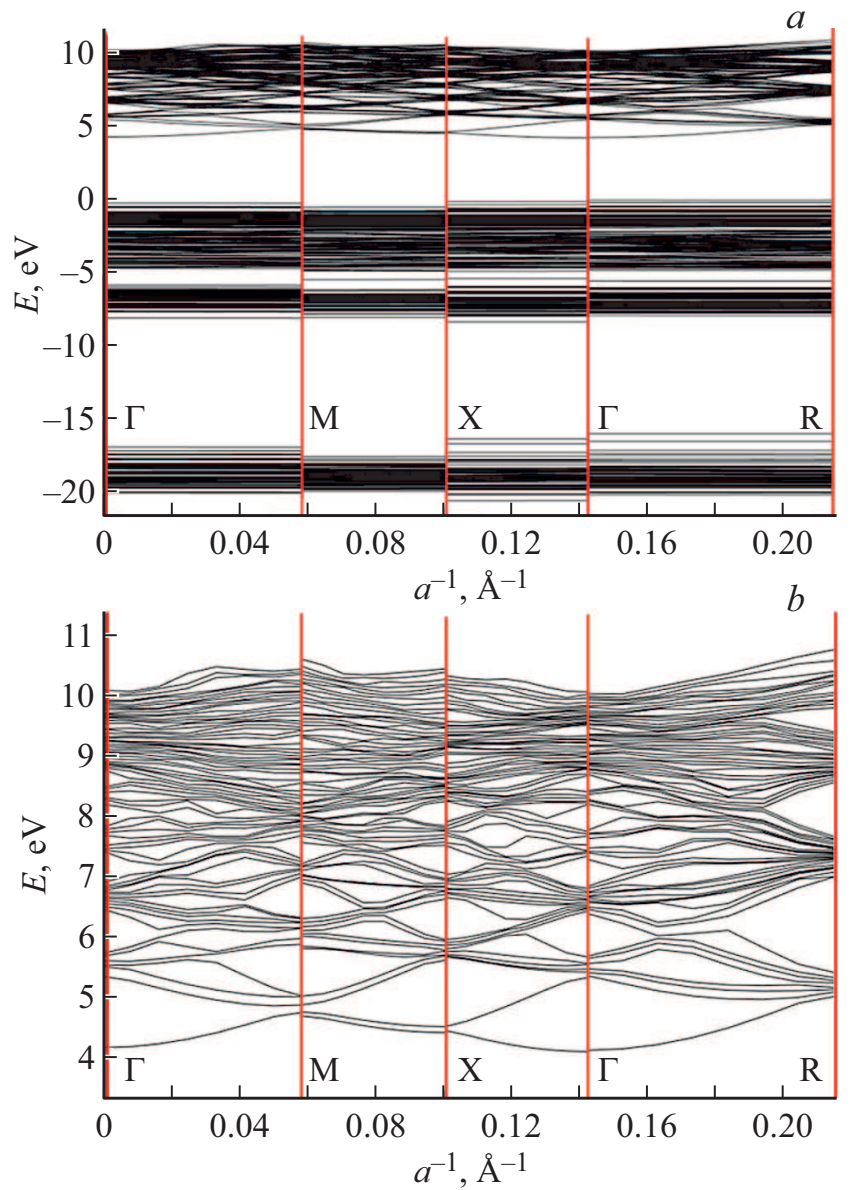

Pис. 4. (Цветной онлайн) Энергетическая зонная структура кристалла гидрата с кубической структурой КС-І с пустыми молекулярными полостями $(a)$. Зона проводимости отдельно представлена на части $(b)$.

Спектр $E(\mathbf{k})$ в области энергий 5-10 eV, что соответствует зоне проводимости, имеет локальные максимумы и минимумы в точках $\Gamma, \mathrm{M}, \mathrm{X}$ и $\mathrm{R}$. Верхняя и нижняя границы зоны проводимости имеют максимумы в точке $\mathrm{R}$ и минимумы в точке Г. Ширина запрещенной зоны в кристалле гидрата КС-I имеет ширину $\Delta E_{G} \approx 5.0 \mathrm{eV}$. Энергия Ферми находится на уровне $E_{F} \approx 2.4 \mathrm{eV}$, что меньше значения для гидрата КС-I с включениями в полости молекул метана $\left(E_{F} \approx 3.0 \mathrm{eV}\right)$.

\section{4. Заключение и выводы}

В настоящей работе представлены результаты крупномасштабного $a b$ initio моделирования гидрата метана с кубической структурой КС-I для широкой области значений температур. Обнаружено хорошее согласие результатов моделирования для плотности и теплоемкости при постоянном объеме с экспериментальными данными [17-19]. На основе анализа плотности электронных состояний определены температурные зависимости электронных свойств гидрата метана: уровень 
энергии Ферми, ширина и границы запрещенной зоны. Установлено, что с увеличением температуры наблюдаются повышения нижней границы зоны проводимости со средней скоростью $d E_{G \max } / d T=0.0053 \mathrm{eV} / \mathrm{K}$, верхней границы валентной зоны со средней скоростью $d E_{G \min } / d T=0.0035 \mathrm{eV} / \mathrm{K}$. В то же время, скорость повышения уровня энергия Ферми составило $d E_{F} / d T=0.0044 \mathrm{eV} / \mathrm{K}$. Рассчитан спектр энергии электронов $E(\mathbf{k})$ вдоль направлений $\mathrm{M}-\mathrm{X}, \mathrm{X}-\Gamma, \Gamma-\mathrm{M}$ и $Г-\mathrm{R}$ для кристалла гидрата с кубической структурой КC-I c пустыми молекулярными полостями. Установлено, что наличие молекул метана приводит к увеличению энергии Ферми гидрата с 2.4 до $3.0 \mathrm{eV}$.

\section{Финансирование работы}

Работа поддержана Российским научным фондом (проект № 19-12-00022). Крупномасштабные молекулярнодинамические расчеты выполнены с использованием оборудования Центра коллективного пользования сверхвысокопроизводительными вычислительными ресурсами МГУ им. М.В. Ломоносова и на вычислительном кластере Казанского (Приволжского) федерального университета.

\section{Конфликт интересов}

Авторы заявляют, что у них нет конфликта интересов.

\section{Список литературы}

[1] E.D. Sloan, C.A. Koh. Clathrate Hydrates of Natural Gases. 3rd rev. ed. CRC Press, Taylor \& Francis USA (2007).

[2] N.J. English, J.M.D. MacElroy. Chem. Eng. Sci. 121, 133 (2015).

[3] R.M. Khusnutdinoff, A.V. Mokshin. J. Non-Cryst. Solids 357, 1677 (2011).

[4] R.M. Khusnutdinoff, A.V. Mokshin. Physica A 391, 2842 (2012).

[5] Р.М. Хуснутдинов. Коллоид. журн. 75, 792 (2013).

[6] R.M. Khusnutdinoff, A.V. Mokshin. J. Cryst. Growth 524, 125182 (2019)

[7] Р.М. Хуснутдинов, А.В. Мокшин. Письма в ЖЭТФ 110, 551 (2019).

[8] R.M. Khusnutdinoff, A.V. Mokshin. ФTT 62, 775 (2020).

[9] P. Guo, Y.-L. Qiu, L.-L. Li, Q. Luo, J.-F. Zhao, Y.-K. Pan. Chin. Phys. B 27, 043103 (2018).

[10] I.-M. Chou, A. Sharma, R.C. Burruss, J. Shu, H.-K. Mao, R.J. Hemley, A.F. Goncharov, L.A. Stern, S.H. Kirby. Proc. Natl. Acad. Sci. USA 97, 13484-7 (2000).

[11] M. Ota, K. Morohashi, Y. Abe, M. Watanabe, J.R.L. Smith, H. Inomata. Energy Convers. Manag. 46, 1680-91 (2005).

[12] M.E. Casco, J. Silvestre-Albero, A.J. Ramirez-Cuesta, F. Rey, J.L. Jorda, A. Bansode, A. Urakawa, I. Peral, M. Martínez-Escandell, K. Kaneko, F. Rodríguez-Reinoso. Nature Commun. 6, 6432 (2015).

[13] G. Kresse, J. Hafner. Phys. Rev. B 47, 558 (1993).

[14] G. Kresse, J. Furthmuller. Phys. Rev. B 54, 11169 (1996).

[15] M. Stackelberg, H.R. Müller. Z. Elktrochem. 58, 25 (1954).
[16] L. Pauling, R.E. Marsh. Proc. Natl. Acad. Sci. USA 38, 112 (1952).

[17] Ю.Ф. Макогон. Геология и полезные ископаемые Мирового океана. № 2, 5 (2010).

[18] W.F. Waite, L.A. Stern, S.H. Kirby, W.J. Winters, D.H. Mason. Geophys. J. Int. 169, 767 (2007).

[19] В.И. Истомин, В.С. Якушев. Газовые гидраты в природных условиях. Недра, М. (1992).

[20] Н. Ашкрофт, Н. Мермин. Физика твердого тела (в двух томах). Мир, М. (1979).

[21] Z. Wang, L. Yang, R. Deng, Z. Yang. arXiv: 1902.10914v1 (2019).

Редактор Ю.Э. Китаев 